\title{
Irregular Pulsating Polarization Dynamics in Gain-Switched Vertical-Cavity Surface-Emitting Lasers
}

\author{
Angel Valle, Marc Sciamanna, and Krassimir Panajotov
}

\begin{abstract}
In this paper, we report on experimental and theoretical investigation on the nonlinear dynamics of the two orthogonal linearly polarized fundamental transverse modes of verticalcavity surface-emitting lasers (VCSELs) under sinusoidal current modulation. Irregular pulses of the power of individual polarizations are measured with a period equal to twice the modulation period. In contrast with individual polarizations, total power displays regular pulsing at twice the modulation period. The variability of pulse streams is characterized by using residence times distributions. We show that the residence time distributions for individual linear polarizations display an exponential decay for large values of that time. Those results are well reproduced by using a theoretical model that includes spontaneous emission fluctuations. However the previous qualitative features remain even in the absence of spontaneous emission noise. Our results therefore suggest that the irregular polarization dynamics have a deterministic origin and can be defined as deterministic chaos.
\end{abstract}

Index Terms-Current modulation, nonlinear dynamics, polarization switching, spontaneous emission noise, vertical-cavity surface-emitting laser (VCSEL).

\section{INTRODUCTION}

$\mathbf{V}$ ERTICAL-CAVITY surface-emitting semiconductor lasers (VCSELs) present significant advantages over their edge-emitting counterparts, including low threshold current, low cost, circular output beam, and easy fabrication in two-dimensional arrays. Although VCSELs are intrinsically single-longitudinal mode devices, emission in multiple transverse and polarization modes is usually found [1]. The polarization is not well fixed and small changes of the injection current or the device temperature may result in a polarization switching (PS) between the two linearly polarized modes. While emission in several transverse modes is usually attributed to spatial-hole burning effects [1]-[4], a number of different

Manuscript received June 11, 2007; revised September 4, 2007. This work was supported in part by the BELSPO IAP $6 / 10$, FWO-Vlaanderen, the OZR-VUB for the GOA and IOF projects, the Conseil Régional de Lorraine, and in part by COST 288 European Action. The work of A.Valle was supported in part by the Ministerio de Educación y Ciencia (MEC) under Project TEC2006-13887-C05-02/TCM.

A. Valle is with the Instituto de Física de Cantabria, CSIC-Universidad de Cantabria, E-39005 Santander, Spain (e-mail: valle@ifca.unican.es).

M. Sciamanna is with the Laboratoire Matériaux Optiques, Photonique et Systèmes (LMOPS), Ecole Supérieure d'Electricité (Supélec), F-57070 Metz, France (e-mail: marc.sciamanna@supelec.fr).

K. Panajotov is with the Department of Applied Physics and Photonics (TWTONA), Vrije Universiteit Brussels, B-1050 Brussels, Belgium. He is also with the Institute of Solid State Physics, 72 Tzarigradsko Chaussee blvd., 1784 Sofia, Bulgaria (e-mail: kpanajotov@tona.vub.ac.be).

Digital Object Identifier 10.1109/JQE.2007.910707 physical mechanisms can be responsible for PS phenomenon in VCSELs. Therefore, different models of PS in VCSELs have been suggested, for example those taking into account spin relaxation mechanisms in semiconductor quantum wells (SFM model) [5], [6], thermal effects [7], or the relative modification of the net modal gain and losses with the injection current [8]-[10].

The study of chaotic behavior in semiconductor lasers is important nowadays from a fundamental point of view and for their potential use for data encryption applications [11]. Current-modulation of laser diodes is a simple and compact way of achieving the chaotic signals needed for those applications [12]. The dynamics of directly modulated semiconductor lasers has received a lot of attention, considering their potential to generate ultrafast sharp pulses but also their rich nonlinear behavior [13]-[22]. Most of existing studies relate to conventional edge-emitting semiconductor lasers [13]-[18]. Just a few reports of chaotic behavior can be found in the literature [15], [16] since only edge-emitting lasers with relatively small gain saturation and spontaneous emission noise parameters might undergo a period doubling route to chaos under current modulation [18]. Studies of nonlinear dynamics in directly modulated VCSELs remain scarce [19]-[22], while being of great interest both for fundamental and applied research [19]-[23]. Some theoretical and experimental work on the dynamics of directly modulated VCSELs with optical feedback has been recently done [24], [25]. The previous studies [19]-[22] are of theoretical nature and, to the best of our knowledge, no experimental study of the nonlinear dynamics of solitary gain-switched VCSELs has been performed yet. Nonlinear dynamics have been theoretically analyzed for linearly polarized single transverse mode [19], [20] and multimode VCSELs [20], [21]. Chaotic behavior appears in the multimode regime due to transverse mode competition [20], [21]. Single-transverse mode VCSELs have an extra degree of freedom with respect to the single-longitudinal mode edge-emitters, the polarization of the emitted light, that can enrich their nonlinear dynamical behavior under current modulation. A recent work has studied the effect the bias current sweep rate on the polarization switching of the directly modulated VCSEL [26]. The nonlinear dynamics of the two orthogonal polarizations of a directly modulated single-transverse mode VCSEL has been recently analyzed from a theoretical point of view [22]. Chaotic dynamics due to polarization competition has been found in a large range of laser and modulation parameters [22]. The chaotic dynamics of the polarization is found for much smaller amplitudes and frequencies of 
modulation than the chaotic dynamics of the transverse modes of the VCSEL. This occurs since the differences in gain/loss between the two polarizations of a given transverse mode are much smaller than gain/loss differences between different transverse modes [22] and then stronger competition between the orthogonal polarizations is obtained.

In this paper we measure the dynamics of the two orthogonal polarizations of a single-transverse mode VCSEL subject to a sinusoidal current modulation to confirm the theoretical predictions of [22]. We have considered several amplitudes and several frequencies in the gigahertz range. For specific values of the modulation frequency we find an irregular pulsed behavior of the power of individual polarizations with a period equal to twice the modulation period. The total power also pulses with the same period but maintaining regularity in the height of the pulses. We define a residence time to characterize the variability of pulse streams. We show that the residence time distributions for individual linear polarizations display an exponential decay for large values of the time. All the previous results are also analyzed from a theoretical point of view by using the spin flip model [6], [22]. Good agreement between theoretical and experimental results is found. Special attention is given to the role played by spontaneous emission fluctuations in the results obtained in this work. We show that, although spontaneous emission noise inclusion is important to obtain a good comparison, the main qualitative results remain in the deterministic model. That indicates that spontaneous emission fluctuations are not essential for explaining our experimental observations. Therefore, the nonlinear dynamics of an essentially deterministic system has been observed.

Our paper is organised as follows. In Section II, we describe our experimental setup and results. In Section III, we present our theoretical model and results. A comparison with the experimental results is also performed in that section. Finally, in Section IV, a brief discussion and a summary of our results are presented.

\section{EXPERIMENTAL RESULTS}

\section{A. Experimental Setup}

Experimentally, the gain-switching of the VCSEL is achieved using the setup presented in Fig. 1. A quantum-well oxide-confined VCSEL supplied by the University of Ulm, emitting around $851 \mathrm{~nm}$, is current modulated by using a Bias-T. The dc current applied to the VCSEL is controlled by a low-noise laser driver. A RF voltage is applied by using a RF-signal generator. The operating VCSEL temperature is also controlled and is set at $26{ }^{\circ} \mathrm{C}$. The beam from the VCSEL is collimated by using a lens (COL). A nonpolarizing 50/50 beamsplitter (BS) splits the beam in two detection branches. In the first one a polarizer (P2) and a power meter (PM) are used to measure sequentally the power emitted by the VCSEL in the two orthogonal polarizations. The second branch is used to perform spectral measurements using either an optical spectrum analyzer (OSA) or a RF spectrum analyzer associated with a photodetector (D1). An optical isolator and a half wave plate were put between the fiber coupler and the P1 polarizer to avoid feedback effects in the VCSEL. Dynamical evolution of the power is recorded with

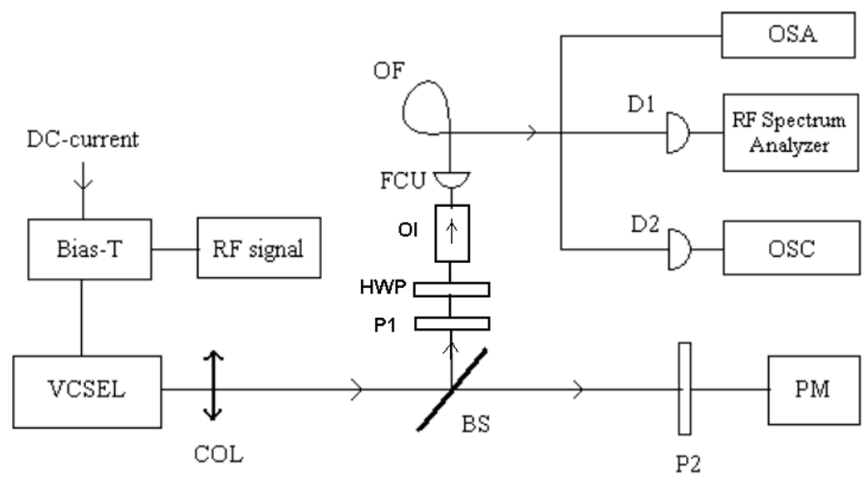

Fig. 1. Experimental setup of gain-switching in VCSEL. COL: collimator; BS beam splitter; P1-P2: polarizers; PM: power meter; HWP: half wave plate; OI: optical isolator; FCU: fiber coupling unit; OF: optical fiber; D1-D2: photodiodes; OSA: optical spectrum analyzer; OSC: oscilloscope.

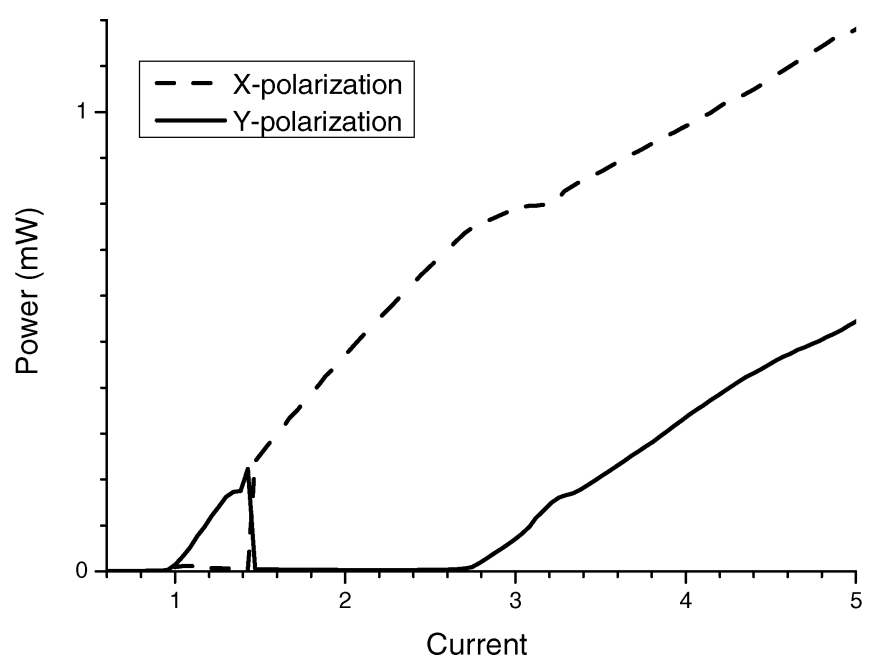

Fig. 2. Polarization-resolved light-current characteristics showing the evolution of the optical power in the horizontal ( $x$, dashed line) and vertical ( $y$, solid line) polarization direction. The current is given in threshold current units.

a digital oscilloscope (OSC) associated with a photodetector (D2). Measurements of the polarized or total power can be performed by inserting or removing the polarizer (P1).

\section{B. Experimental Results}

The polarization-resolved light-current characteristic of the free-running VCSEL is presented in Fig. 2. If the bias current is increased the VCSEL first emits a fundamental linearly polarized vertical mode ( $y$-LP mode) with a threshold current, $I_{\mathrm{th}}$, of $1.2 \mathrm{~mA}$. As the injection current is increased a polarization switching (PS) between the orthogonal fundamental modes is found at around $1.4 I_{\mathrm{th}}$. That switching is of type I (PS I) because it occurs from the high-frequency mode ( $y$-LP mode) to the mode with a lower frequency ( $x$-LP mode). The VCSEL exhibits a single-transverse mode operation for bias currents less than $2.7 I_{\mathrm{th}}$. For higher currents, excitation of the first higher order mode is observed.

After characterising the continuous-wave $(\mathrm{CW})$ polarization behavior of the VCSEL a sinusoidal modulation of the current is applied. The modulation frequency is around $1 \mathrm{GHz}$. The dc bias current has been chosen slighty smaller than the current at which PS occurs. Amplitudes of the modulation are 

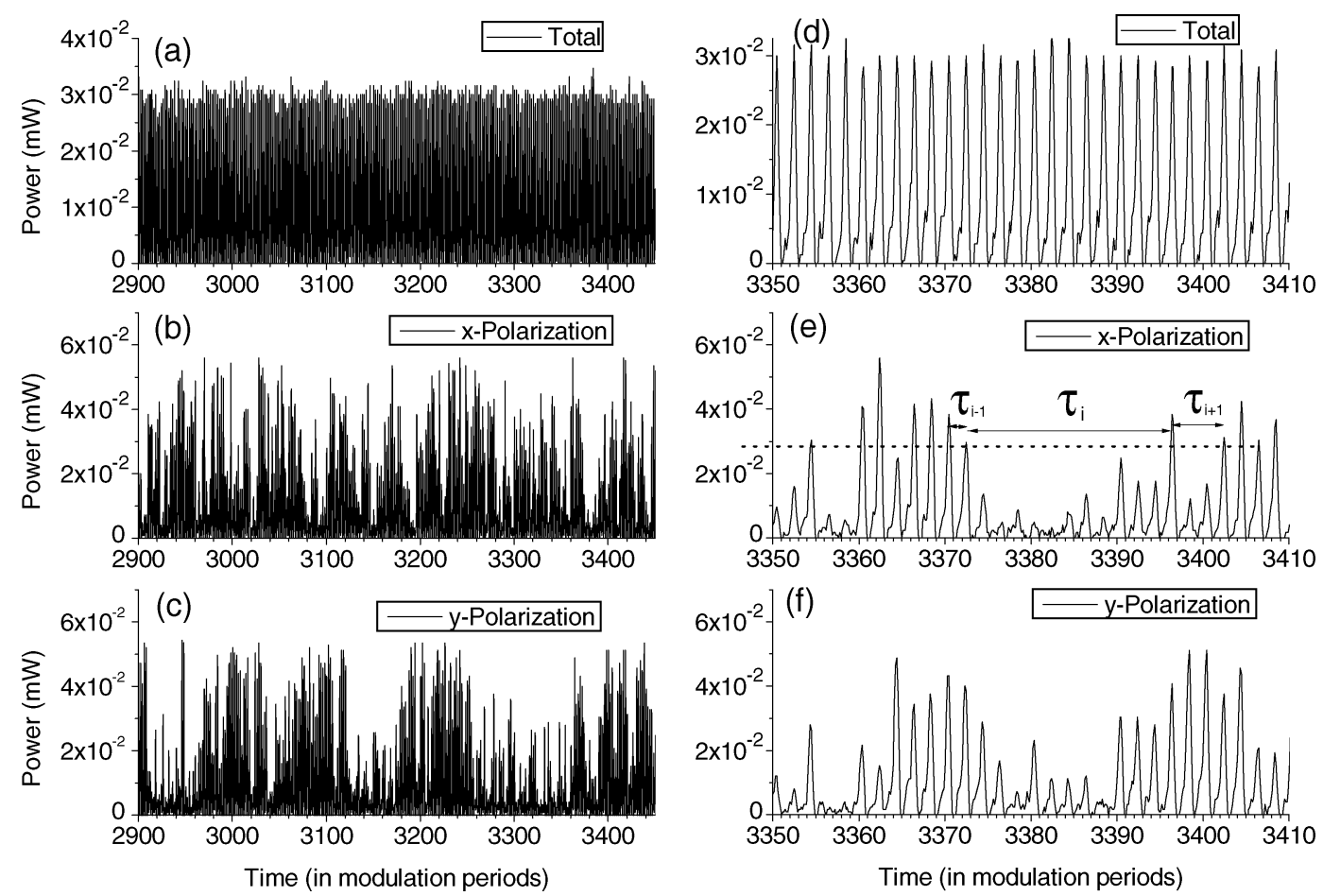

Fig. 3. Experimental time traces of the intensities of the total, $x$-polarized and $y$-polarized powers. Results plotted in (d)-(f) correspond to zooms of (a)-(c), respectively, where the latter result from three independent experimental runs. The current modulation is such that $f_{m}=2.88 \mathrm{GHz}, I_{\mathrm{dc}}=1.26 I_{\mathrm{th}}$, and $\Delta I=$ $0.89 I_{\text {th }}$.

chosen large enough to achieve gain-switching operation (the VCSEL current always spends a fraction of the period below the threshold current) and small enough to avoid the excitation of the higher order transverse mode. Repetitive gain-switched pulses (one pulse each modulation period $T$ ) are obtained for small modulation frequencies. A regular stream of pulses with similar heights is obtained for the total power. The behavior of individual polarizations is different: again a pulse of a given polarization appears each modulation period but its height largely fluctuates from one pulse to another. Pulses in both polarizations fluctuate in such a way that the total power remains regularly pulsing with pulses of similar heights. That situation indicates a clear anticorrelation between both polarizations. For large enough amplitudes of modulation the laser current goes well below the threshold value in such a way that the laser power reaches the spontaneous emission noise levels. The polarization that is excited with larger power in each modulation period is mainly determined by the spontaneous emission noise events occuring just before the switch-on of the pulse since the gain/losses differences between polarizations are very small. Then for small modulation frequencies spontaneous emission noise is essential in determining the dynamics of the polarization in the pulsed VCSEL.

More interesting nonlinear dynamics is found by increasing the modulation frequency. We show in Fig. 3 the temporal traces of the total and polarized powers when the modulation frequency $f_{m}=1 / T$ is $2.88 \mathrm{GHz}$, the dc current $I_{\mathrm{dc}}$ is 1.26 $I_{\mathrm{th}}$, and the current amplitude $\Delta I$ is $0.89 I_{\mathrm{th}}$. The influence of a higher order transverse mode is very weak because the optical spectrum under those modulation conditions show that the supression ratio was $30 \mathrm{~dB}$. The right column of that figure is composed of zooms of the left part to better appreciate the details of the pulses. A regular stream of pulses is found for the total power. Those pulses have similar heights and appear each two periods of modulation [see Fig. 3(d)]. Small shoulders appear that are reminiscences of the period- 1 solution. When the amplitude of the modulation decreases those shoulders become larger until similar heights are obtained for all the pulses in such a way that only one pulse appears each modulation cycle (period-1 solution). Pulses in individual polarizations also appear with that periodicity but their heights are very irregular. Fig. 3(b) and (c) shows that there are wide temporal regions in which pulses in one polarization are very small (see for instance, Fig. 3(c) for times between 3120 and 3170 modulation periods). For those regions pulses in the orthogonal polarization have similar heights. This fact is deduced from the regularity of the total power and can not be deduced by directly comparing Fig. 3(b) and (c) because the traces shown in Fig. 3(a)-(c) have been obtained for different runs of the experiment. Due to technical constraints we can not record the time traces of the two orthogonal linear polarizations simultaneously.

One way of characterizing the irregularity of the streams of pulses is by measuring the duration of the regions where one polarization pulsates at $2 \mathrm{~T}$ with large power and the other pulsates with the same periodicity but with small power. We estimate that duration by using the residence time $\tau$ that we define as follows. First, we fix a power level that we choose as one half of the maximum power of the complete temporal series. Then $\tau$ is given by the time between consecutive crossings, from below to above, of that level. The process for obtaining the residence times, together with some values of that random variable, $\tau_{i}$, are illustrated in Fig. 3(e). We have acquired data over a $0.4-\mathrm{ms}$ window 

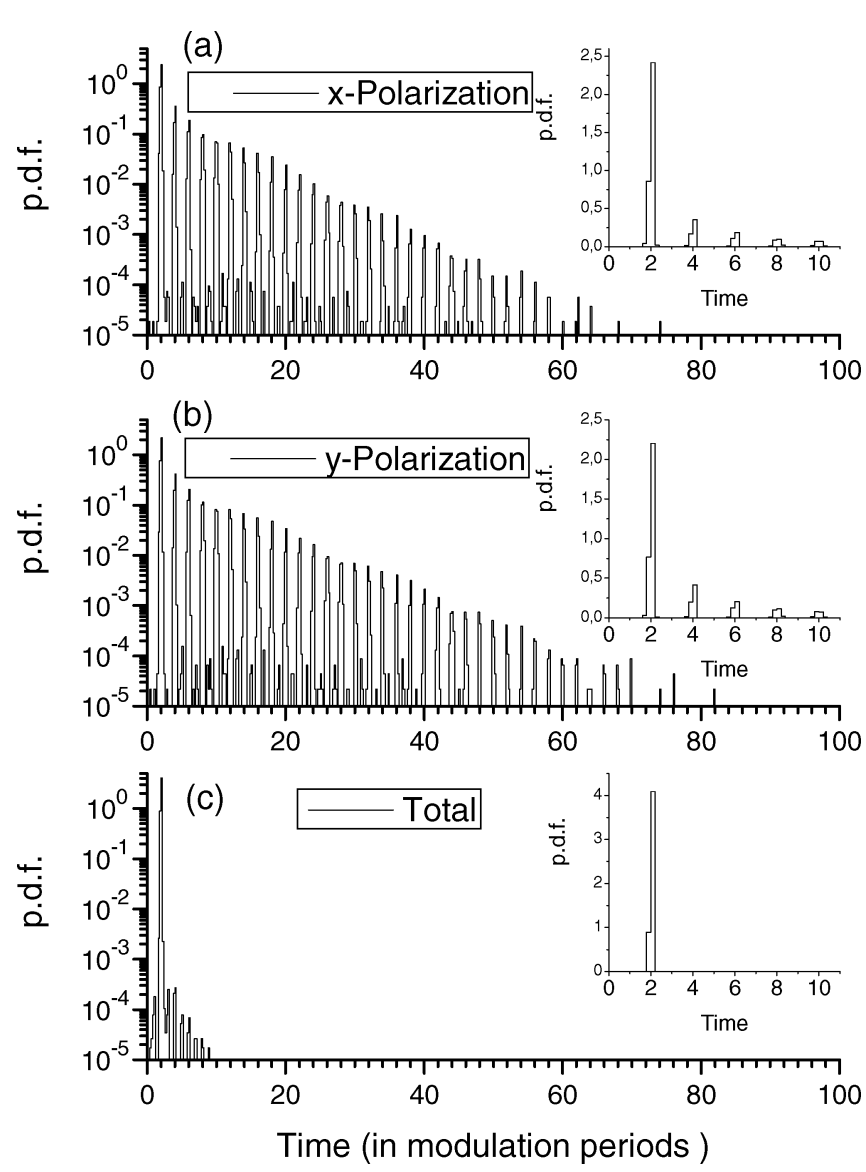

Fig. 4. Experimental probability density functions of the residence time for the (a) $x$-polarization, (b) $y$-polarization and (c) total power. Insets represent zooms of the small $\tau$ region. The parameters of the modulation are those of Fig. 3.

with a 50-ps sampling time. The probability density functions of the residence times for the individual polarizations and the total power are shown in Fig. 4. The distributions for the two polarizations are very similar: they both present a multipeaked structure with a long exponential envelope. Distributions have been plotted in a logarithmic scale to show that exponential decay of the pulse envelope. A modulation seems to be superimposed on that exponential decay. Peaks appear at multiples of $2 \mathrm{~T}$ since that is precisely the periodicity of the pulses. We also show in Fig. 4 zooms of the regions of small $\tau$ in a linear scale to better appreciate the structure of those peaks. The width of the peaks is related to the uncertainty of the time at which the pulse crosses the power threshold. The long exponential tail is a signature of the variability of the stream of pulses. This is in contrast with the absence of that tail when looking at the distribution of $\tau$ for the total power [see Fig. 4(c)]. That absence is explained in terms of the regular behavior displayed by the time traces of the total power.

The irregular behavior of individual polarizations in contrast to the regular one of the total power can also be illustrated by looking at the corresponding RF spectra. Those spectra are shown in Fig. 5. A large peak appears at $f_{m} / 2$ for the individual polarizations and the total power. Those peaks are an indication of the period-doubling dynamics observed in the time series. The large pedestal that appears around the peaks of the spectra

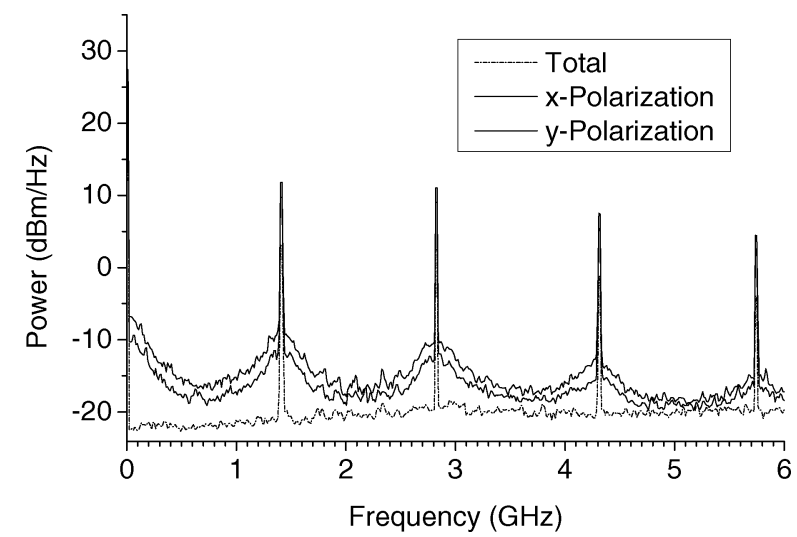

Fig. 5. Experimental RF spectra of the total, $x$-polarized and $y$-polarized power. The parameters of the modulation are those of Fig. 3 .

of individual polarizations is an indication of their irregular dynamics. RF power levels at low frequencies are much larger for the individual polarizations than for the total power, as a result of the anticorrelation between both polarizations. Similar qualitative behaviors were found in a $\pm 0.2-\mathrm{GHz}$ range. The $2.88-\mathrm{GHz}$ modulation frequency value has been chosen since the peak that indicates period-doubling in the RF spectrum of the total power was maximum at that frequency.

\section{ThEORETICAL RESULTS}

\section{A. Theoretical Model}

In this work we consider a model for the polarization of a single-transverse mode VCSEL [6], [22] that takes into account the combined effect of the VCSEL anisotropies, the linewidth enhancement factor and the spin-flip relaxation processes within a framework known as the SFM model [5]. The equations for the left and right circularly polarized components of the slowly varying optical field, $E_{ \pm}$, the total population difference between conduction and valence bands, $N$, and the difference between the two distinct subpopulation inversion densities which couple separately to the emission of left and right circularly polarized light, $n$, are the following:

$$
\begin{aligned}
\dot{E}_{ \pm}= & \kappa(1+i \alpha)[(N \pm n)-1] E_{ \pm}-\left(\gamma_{a}+i \gamma_{p}\right) E_{\mp} \\
& +\sqrt{\beta_{\mathrm{sp}}(N \pm n)} \xi_{ \pm} \\
\dot{N}= & -\gamma\left[N-I+(N+n)\left|E_{+}\right|^{2}+(N-n)\left|E_{-}\right|^{2}\right] \\
\dot{n}= & -\gamma_{s} n-\gamma\left[(N+n)\left|E_{+}\right|^{2}-(N-n)\left|E_{-}\right|^{2}\right]
\end{aligned}
$$

where $I$ is the normalized injection current (its value at threshold is $I_{\mathrm{th}}=1$ ), modulated according to $I=$ $I_{\mathrm{dc}}+\Delta I \sin \left(2 \pi f_{m} t\right) . \kappa$ is the electric field decay rate that includes the internal and facet losses, $\alpha$ is the alpha factor or linewidth enhancement factor that describes phase-amplitude coupling mechanisms in semiconductor lasers and $\gamma$ is the carrier relaxation rate. $\gamma_{s}=\gamma+2 \gamma_{j}$, where $\gamma_{j}$ is a coupling rate between the two circularly polarized radiation channels, which models different microscopic relaxation mechanisms that equilibrate the spin of carriers [5]. $\gamma_{p}\left(\gamma_{a}\right)$ is the magnitude of linear birefringence (dichroism), per intracavity round-trip 

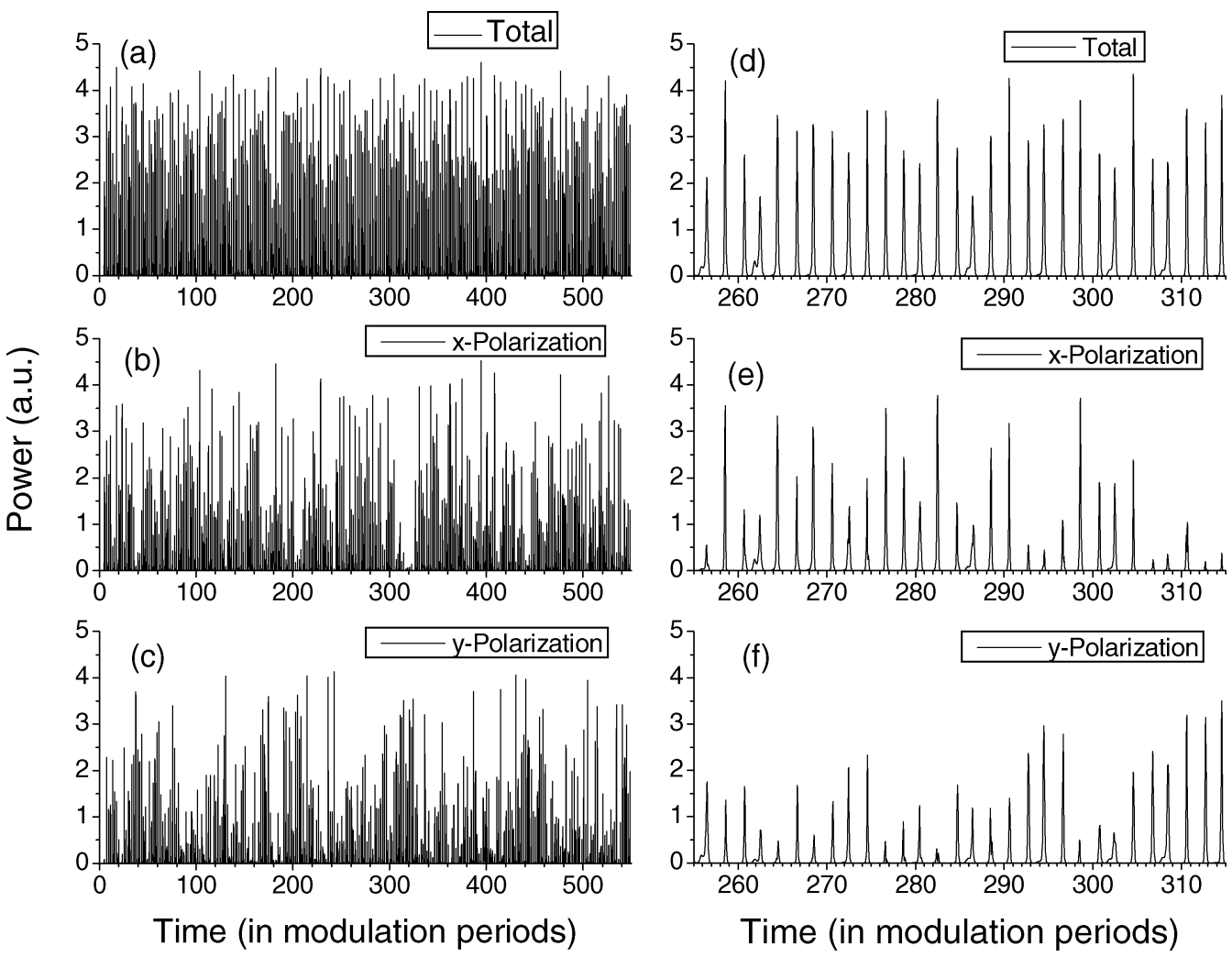

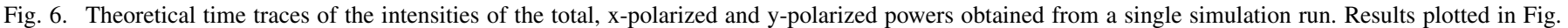
6(d)-(f) and correspond to zooms of Fig. 6(a)-(c), respectively. The current modulation is such that $f_{m}=2.88 \mathrm{GHz}, I_{\mathrm{dc}}=1.3$, and $\Delta I=0.7$.

time. $\beta_{\mathrm{sp}}$ is the spontaneous emission rate and $\xi_{ \pm}$are Gaussian white noises of zero mean value and delta-correlated in time.

\section{B. Theoretical Results}

We first calculate the polarization-resolved light-current characteristic of the free-running VCSEL. We solve the (1)-(3) by using the numerical algorithm reported in [27] with a 0.01 ps integration time step. The laser parameters have been chosen for obtaining a similar light-current characteristic to the experimental one presented in Fig. 2. A PS I switching is obtained at a current of 1.3 when $\kappa=300 \mathrm{~ns}^{-1}, \alpha=3, \gamma=1 \mathrm{~ns}^{-1}$, $\gamma_{s}=50 \mathrm{~ns}^{-1}, \gamma_{a}=0.1 \mathrm{~ns}^{-1}, \gamma_{p}=10 \mathrm{~ns}^{-1}$. We then calculate the time traces of the total and polarized powers for a sinusoidal modulation of the current with a frequency equal to the experimental one $\left(f_{m}=2.88 \mathrm{GHz}\right)$. The distributions of the residence times are also calculated for a variety of $I_{\mathrm{dc}}$, $\Delta I$ and $\beta_{\mathrm{sp}}$ for finding the better match with the experimental results. The best agreement is found when $I_{\mathrm{dc}}=1.3$ and $\Delta I=0.7$, that are near to the experimental values used in the previous section, and $\beta_{\mathrm{sp}}=10^{-4}$. The temporal traces of the total and polarized powers for those parameters are shown in Fig. 6. Nonlinear dynamics similar to the experimentally reported in the previous section is found. Irregular pulses of the power of individual polarizations are obtained with a period equal to twice the modulation period while the total power displays a more regular pulsing with the same periodicity. We note that the pulsing in the total power is not as regular as the experimental one. We also note that peaks in the simulation are narrower than the experimental ones. We explain it in terms of the absence of gain staturation terms in the spin-flip-model. In contrast with the experimental results reported in Fig. 3, the anticorrelation between both polarizations is now clearly seen by comparing Fig. 6(b) and (c) [or Fig. 6(e) and (f)] because all the traces shown in Fig. 6 have been obtained for the same simulation run.

In the previous section we characterized the variability of the streams of pulses by measuring the residence time, $\tau$, distribution. The theoretical probability density functions of $\tau$ for the individual polarizations and the total power are shown in Fig. 7. Those distributions have been calculated by using data obtained over a 0.4-ms temporal window, equal to the experimentally used. The residence time distributions for the two polarizations present the same qualitative features as the experimental ones of Fig. 4, namely, the multipeaked structure at multiples of $2 \mathrm{~T}$ and the long exponential tail. Also, the distribution of $\tau$ for the total power is concentrated at low values of that time because of the regular behavior of the total power. The residence time distributions change when changing the modulation parameters. The increase of $\Delta I$, for a fixed value of $I_{\mathrm{dc}}$, change the exponential tail of the distribution corresponding to the $y$-polarization in a significant way (the maximum value changes from 32 to 96 modulation periods when $\Delta I$ changes from 0.6 to 0.8 , for $I_{\mathrm{dc}}=1.3$ ). The tails corresponding to the $x$-polarization and the total power remain unchanged. The increase of $I_{\mathrm{dc}}$, for a fixed value of $\Delta I$, change the exponentials tails of the RTDs 

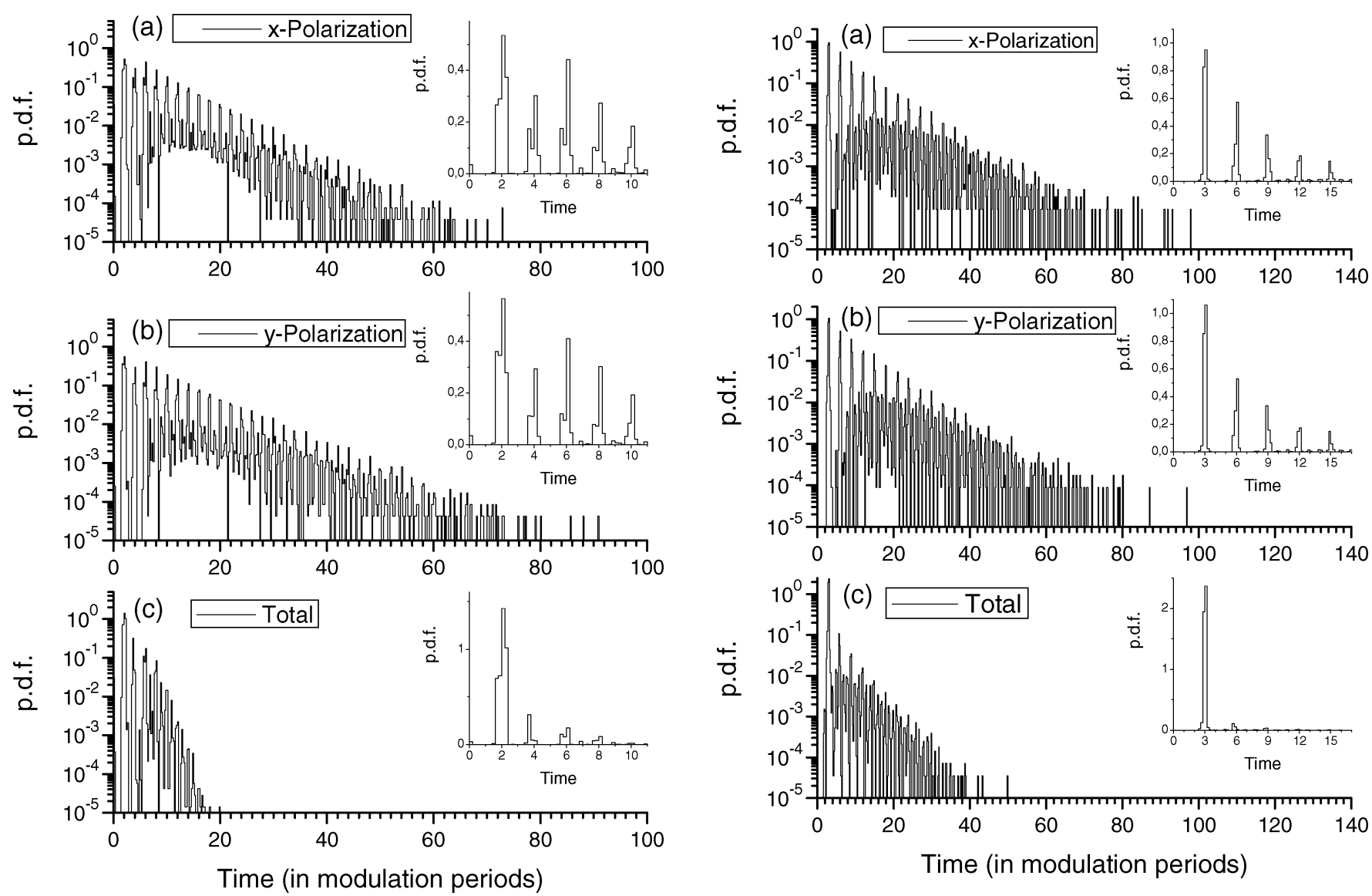

Fig. 7. Theoretical probability density functions of the residence time for the (a) $x$-polarization, (b) $y$-polarization and (c) total power. Insets represent zooms of the small $\tau$ region. The parameters of the modulation are those of Fig. 6 .

corresponding to both polarizations in such a way that the maximum residence times decrease when increasing $I_{\mathrm{dc}}$.

We have also found different dynamical behaviors in which irregular pulses of the power of individual polarizations are obtained with a period equal to $3 \mathrm{~T}$ while the total power displays regular pulsing with a $3 \mathrm{~T}$ period. We show in Fig. 8 the residence time distributions obtained when changing $I_{\mathrm{dc}}, \Delta I$, and $\beta_{\mathrm{sp}}$. Multiple peaks appear at multiples of $3 \mathrm{~T}$ in the individual polarizations while the probability for $\tau$ corresponding to the total power is concentrated at the $3 \mathrm{~T}$ value. Again the slow exponential decay is clear for the $\tau$ that corresponds to the individual polarizations. The laser parameters have changed (a very different value of $\beta_{\mathrm{sp}}$ is considered) with respect to the case of Figs. 6 and 7. That can be the reason why that behavior has not been found experimentally.

\section{DISCUSSION AND CONCLUSION}

Our theoretical results of the previous section have been obtained with a model that includes a random spontaneous emission term. It is desirable to know if the behavior that we have found, especially the slow exponential decay of the residence time distributions, is caused by the fluctuating nature of the spontaneous emission or if it is just a result inherent to the deterministic dynamics of the system. To answer that question we have repeated the simulations reported in Figs. 6 and 7 with

Fig. 8. Theoretical probability density functions of the residence time for the (a) $x$-polarization, (b) $y$-polarization and (c) total power. Insets represent zooms of the small $\tau$ region. The parameters of the simulation are $f_{m}=2.88 \mathrm{GHz}$, $I_{\mathrm{dc}}=1.22, \Delta I=0.9$, and $\beta_{\mathrm{sp}}=10^{-6}$.

$\beta_{\mathrm{sp}}=0$. In those deterministic simulations we have found the same qualitative dynamics as in the simulations with included spontaneous emission noise. The driving force for the fluctuations in the deterministic simulations is the complicated nonlinear dynamics of the system. We show in Fig. 9 the distributions of the residence times for the deterministic case. They remain qualitatively similar to those found when spontaneous emission noise is included (see Fig. 7). We only find changes in the extension of the exponential decay: the maximum residence times for the $x$ and $y$ polarizations are 80 and $150 \mathrm{~T}$, respectively, in the deterministic simulations. That can be explained by taking a look in Fig. 10 at the temporal traces of the polarized intensities obtained with the simulation including noise $\left(\beta_{\mathrm{sp}}=10^{-4}\right)$. In Fig. 10, the intensities are plotted in logarithmic scale to show the effect of spontaneous emission noise and the parameters are the same than those of Fig. 6. Fig. 10 shows that the minimum values of the individual polarizations are above the power level where the fluctuations due to spontaneous emission dominate the evolution (around $10^{-7}$ ).

To conclude, we have performed a theoretical and an experimental study of the nonlinear dynamics of the two orthogonal linearly polarized fundamental transverse modes of VCSELs under sinusoidal current modulation. For large modulation frequencies, around $2.9 \mathrm{GHz}$, we have found irregular pulses of the power of individual polarizations with a period equal to twice the modulation period while the total power displays regular 

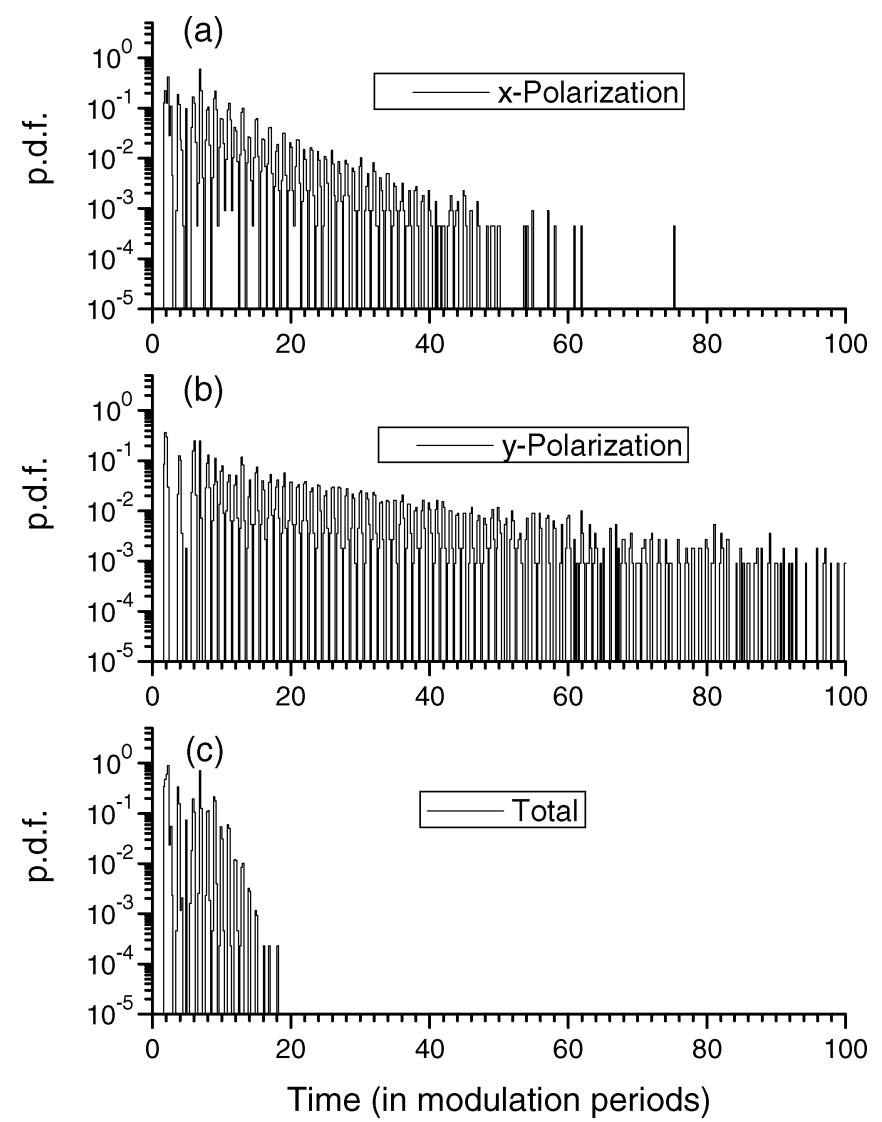

Fig. 9. Theoretical probability density functions of the residence time for the (a) $x$-polarization, (b) $y$-polarization and (c) total power. The parameters are those of Fig. 6 but with $\beta_{\mathrm{sp}}=0$.

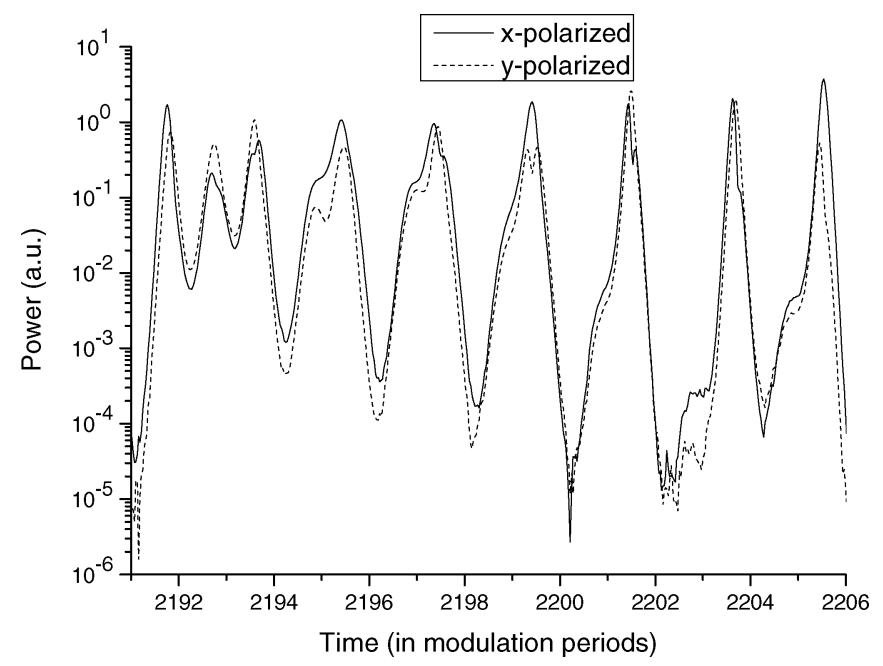

Fig. 10. Theoretical time traces of the $x$-polarized and $y$-polarized powers. The parameters are such that $f_{m}=2.88 \mathrm{GHz}, I_{\mathrm{dc}}=1.3, \Delta I=0.7$ and $\beta_{\mathrm{sp}}=10^{-4}$.

pulsing at twice the modulation period. By changing the simulation parameters we have also found a situation where irregular pulses of the power of individual polarizations appear with a period equal to three times the modulation period while the total power displays regular pulsing at three times the modulation period. The analysis of the influence of the laser parameters on the dynamics reported in this work is interesting and will be the subject of future work. The variability of pulse streams has been characterized by using residence times probability density functions. We have shown that those distributions for individual linear polarizations display an exponential decay of pulse envelope for large values of the time. Those results are well reproduced by using a theoretical model that includes spontaneous emission fluctuations. However that qualitative features remain even in the absence of spontaneous emission noise. Our results therefore suggest that the irregular polarization dynamics have a deterministic origin and can be defined as deterministic chaos.

In our experimental work we have focused on very specific values of the system parameters: just one value of the modulation frequency, dc bias current and amplitude of the modulation for which the more complicated dynamics was observed. The theoretical work have tried to identify the parameters of the model that can explain that behavior.That irregular behavior is then a particular case of the chaotic deterministic dynamics due to polarization competition that was described in [22]. An experimental analysis of the dynamics for a large range of laser and modulation parameters would be also very interesting for comparing with all the predictions of [22] and it will be the subject of future work.

\section{ACKNOWLEDGMENT}

The authors would like to thank R. Michalzik and his coworkers at the Optoelectronics Department, University of Ulm, Ulm, Germany, for providing the VCSELs. The authors also acknowledge T. Iwanow and M. Arizaleta for their help in the experiments.

\section{REFERENCES}

[1] C. J. Chang-Hasnain, J. P. Harbison, G. Hasnain, A. C. von Lehmen, L. T. Florez, and N. G. Stoffel, "Dynamic, polarization and transverse mode characteristics of vertical cavity surface emitting lasers," IEEE J. Quantum Electron., vol. 27, no. 6, pp. 1402-1409, Jun. 1991.

[2] D. Vakhshoori, J. D. Wynn, G. J. Zydzik, M. Asom, K. Kojima, R. E. Leibenguth, and R. A. Morgan, "Top-surface emitting lasers with $1.9 \mathrm{~V}$ threshold voltage and the effect of spatial hole burning on their transverse mode operation and efficiencies," Appl. Phys. Lett., vol. 62, no. 13, pp. 1448-1450, 1993.

[3] O. Buccafusca, J. L. A. Chilla, J. J. Rocca, S. Feld, C. Wilmsen, V. Morozov, and R. E. Leibenguth, "Transverse mode dynamics in vertical cavity surface emitting lasers excited by fast electrical pulses," Appl. Phys. Lett., vol. 68, no. 5, pp. 590-592, 1996.

[4] A. Valle, J. Sarma, and K. A. Shore, "Spatial hole burning effects on the dynamics of vertical-cavity surface-emitting laser diodes," IEEE J. Quantum Electron., vol. 31, no. 8, pp. 1423-1431, Aug. 1995.

[5] M. San Miguel, Q. Feng, and J. V. Moloney, "Light-polarization dynamics in surface-emitting semiconductor lasers," Phys. Rev. A, vol. 52, no. 2, pp. 1728-1739, 1995.

[6] J. M. Martín-Regalado, F. Prati, M. San Miguel, and N. B. Abraham, "Polarization properties of vertical cavity surface-emitting lasers," IEEE J. Quantum Electron., vol. 33, no. 5, pp. 765-783, May 1997.

[7] K. D. Choquette, R. P. Schneider, K. L. Lear, and R. E. Leibenguth, "Gain-dependent polarization properties of vertical-cavity lasers," IEEE J. Select. Topics Quantum Electron., vol. 1, no. 2, pp. 661-666, Mar./Apr. 1995.

[8] K. Panajotov, B. Ryvkin, J. Danckaert, M. Peeters, H. Thienpont, and I. Veretennicoff, "Polarization switching in VCSELs due to thermal lensing," IEEE Photon. Technol. Lett., vol. 10, no. 1, pp. 6-8, Jan. 1998.

[9] A. Valle, K. A. Shore, and L. Pesquera, "Polarization selection in birefringent vertical-cavity surface emitting lasers," J. Lightw. Technol, vol. 14, no. 9, pp. 2062-2068, Sep. 1996. 
[10] B. Ryvkin, K. Panajotov, A. Georgievski, J. Danckaert, M. Peeters, G. Verschaffelt, H. Thienpont, and I. Veretennicoff, "Effect of photonenergy-dependent loss and gain mechanisms on polarization switching in vertical-cavity surface-emitting lasers," J. Opt. Soc. Amer. B, vol. 16, pp. 2106-2113, 1999.

[11] A. Ergyris, D. Syvridis, L. Larger, V. Annovazzi-Lodi, P. Colet, I. Fischer, J. Garcia-Ojalvo, C. R. Mirasso, L. Pesquera, and K. A. Shore, "Chaos based-communications at high bit rates using commercial fiberoptic links," Nature, vol. 438, no. 7066, pp. 343-346, 2005.

[12] R. P. Walker, P. Rees, I. Pierce, P. S. Spencer, and G. W. Roberts, "Analysis of chaos generated by a modulated self-pulsating laser diode," IEE Proc. Optoelectron., vol. 152, no. 2, pp. 90-96, 2005.

[13] C. H. Lee, T. H. Yoon, and S. Y. Shin, "Period doubling and chaos in a directly modulated laserd diode," Appl. Phys. Lett., vol. 46, no. 1, pp. 95-97, 1985.

[14] Y. C. Chen, H. G. Winful, and J. M. Liu, "Subharmonic bifurcations and irregular pulsing behavior of modulated semiconductor-lasers," Appl. Phys. Lett., vol. 47, no. 3, pp. 208-210, 1985.

[15] H. F. Liu and W. F. Ngai, "Nonlinear dynamics of a directly modulated $1.55 \mu \mathrm{m}$ InGaAsP distributed feedback laser," IEEE J. Quantum Electron., vol. 29, no. 6, pp. 1668-1675, Jun. 1993.

[16] Y. Matsui, S. Kutsuzawa, S. Arahira, Y. Ogawa, and A. Suzuki, "Bifurcation in 20-GHz gain-switched $1.55 \mu \mathrm{m} \mathrm{MQW} \mathrm{lasers} \mathrm{and} \mathrm{its} \mathrm{control}$ by CW injection seeding," IEEE J. Quantum Electron., vol. 34, no. 3, pp. 1213-1223, 1998.

[17] H. Lamela, G. Carpintero, and P. Acebo, "Period tripling and chaos in the dynamic behavior of directly modulated diode lasers," IEEE J. Quantum Electron., vol. 34, no. 3, pp. 1797-1801, Mar. 1998.

[18] C. Mayol, R. Toral, C. R. Mirasso, S. I. Turovets, and L. Pesquera, "Theory of main resonances in directly modulated diode lasers," IEEE J. Quantum Electron., vol. 38, no. 3, pp. 260-269, Mar. 2002.

[19] S. F. Yu, "Nonlinear dynamics of vertical-cavity surface-emitting lasers," IEEE J. Quantum Electron., vol. 35, no. 3, pp. 332-341, Mar. 1999.

[20] A. Valle, L. Pesquera, S. I. Turovets, and J. M. López, "Nonlinear dynamics of current modulated vertical-cavity surface-emitting lasers," Opt. Commun., vol. 208, pp. 173-182, 2002.

[21] J. Y. Law and G. P. Agrawal, "Nonlinear spatio-temporal dynamics due to transverse mode competition in gain-switched microcavity semiconductor lasers," Opt. Commun., vol. 138, pp. 95-98, 1997.

[22] M. Sciamanna, A. Valle, P. Megret, M. Blondel, and K. Panajotov, "Nonlinear polarization dynamics in directly modulated vertical-cavity surface-emitting lasers," Phys. Rev. E, vol. 68, p. 016207, 2003.

[23] K. Iga, "Surface-emitting laser: Its birth and generation of new optoelectronics field," IEEE J. Sel. Top. Quantum Electron., vol. 6, no. 6, pp. 1201-1215, Nov./Dec. 2000.

[24] M. S. Torre, C. Masoller, P. Mandel, and K. A. Shore, "Transversemode dynamics in directly modulated vertical-cavity surface-emitting lasers with optical feedback," IEEE J. Quantum Electron., vol. 40, no. 6, pp. 620-628, Jun. 2004.

[25] Y. Hong, S. Bandyopadhyay, and K. A. Shore, "Spectral signatures of the dynamics of current-modulated vertical-cavity surface-emitting lasers subject to optical feedback," J. Opt. Soc. Amer. B, vol. 22, no. 11, pp. 2350-2356, 2005

[26] J. Paul, C. Masoller, Y. H. Hong, P. S. Spencer, and K. A. Shore, "Experimental study of polarization switching of vertical-cavity surface-emitting lasers as a dynamical bifurcation," Opt. Lett., vol. 31, no. 6, pp. 748-750, 2006.

[27] J. M. Sancho, M. San Miguel, S. L. Katz, and J. D. Gunton, "Analytical and numerical studies of multiplicative noise," Phys Rev. A, vol. 26, no. 3, pp. 1589-1609, 1982.

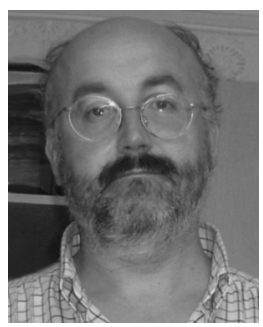

Angel Valle was born in Reinosa, Cantabria, Spain, in 1965. He received the M.Sc. and Ph.D. degrees in physics from the Universidad de Cantabria, Cantabria, Spain, in 1988 and 1993, respectively.

During 1994 and 1995, he was a Postdoctoral Fellow at the School of Electronic and Electrical Engineering, University of Bath, Bath, U.K. In 1996, he joined the Instituto de Física de Cantabria (CSIC-UC), Cantabria, Spain. Since 1998, he has been Lecturer at the Departamento de Física Moderna, University of Cantabria, Spain. His research interests are in the areas of vertical-cavity surface-emitting lasers, noise, and nonlinear dynamics of semiconductor lasers.

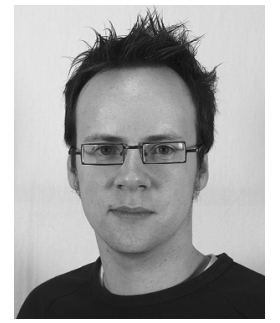

Marc Sciamanna graduated in electrical engineering from the Faculté Polytechnique de Mons, Belgium, in 2000. He received the Ph.D. degree from Faculté Polytechnique de Mons, as a Research Fellow of the "Fonds National de la Recherche Scientifique" (FNRS). His Ph.D. dissertation was entitled "Nonlinear dynamics and polarization properties of externally driven semiconductor lasers."

Currently, he is a permanent Researcher, Lecturer and faculty staff member of SUPELEC (Ecole Supérieure d'Electricité), Metz, France, and LMOPS CNRS UMR-7132 Laboratory, Metz, France. His research interests include the physics and nonlinear dynamics of semiconductor lasers, the polarization properties of VCSELs, the study of dynamical instabilities related to optical feedback, optical injection, or large current modulation, synchronization and chaotic encryption using laser diodes. He is author of about 50 research papers in international journals and conference proceedings. He has given several invited talks in international conferences and has worked as a scientific program committee member in SPIE Photonics Europe 2006. He organized and cochaired the PHASE international workshop (PHysics and Applications of SEmiconductor LASERs), Supélec, Metz, France, in 2005; and acted as a Guest Editor of a special issue of Optical and Quantum Electronics related to the PHASE workshop.

Dr. Sciamanna acted as a Board Member of the IEEE/LEOS Benelux Chapter, co-founded the IEEE/LEOS Benelux Student Chapter and co-chaired the IEEE/LEOS Workshop on Low-Cost Photonics (Mons, Belgium, June 13, 2003). He is Vice-Chairman of working group 2 "Physics of Devices" in European Action COST 288. He has been awarded with the Prize for Best Engineer in Electricity from Faculté Polytechnique de Mons (2000), the OSA-Newfocus student travel grant award (2002), the IEEE/LEOS Graduate Student Fellowship Award (2002), and the SPIE F-MADE Scholarship Award in Optical Engineering (2003).

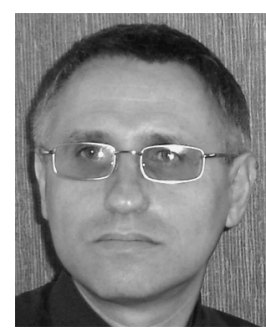

Krassimir Panajotov received the B.S., Ph.D., and D.Sc. degrees in physics from Sofia University, Sofia, Bulgaria, in 1982, 1988, and 2002, respectively. His $\mathrm{Ph} . \mathrm{D}$. dissertation was in the field of nonlinear optics and optical bistability. His D.Sc. thesis is about polarization of light as a mechanism for switching and control in photonics and contains experimental and theoretical results on nonlinear bistable devices, fiber-optic devices, and vertical-cavity surface-emitting lasers (VCSELs).

Since 1982, he has been appointed at the Institute of Solid State Physics (ISSP), Bulgarian Academy of Sciences, currently as Professor. Since 2001, he has lead the group on Micro- and Nano Photonics at ISSP. During 1996-1998, he was a Guest Researcher at the Vrije Universiteit Brussels (VUB), Bruseels, Belgium, working on the topic of polarization switching in VCSELs. In 2000, he again joined VUB as a Guest Professor His research activities are in thin film and fiber optics, nonlinear optics, semiconductor lasers, photonic crystal devices, and nonlinear laser dynamics in edge-emitting lasers and VCSELs. In these fields, he holds more than 90 SCI-stated journal papers and more than 90 publications in international conference proceedings. He was an invited speaker at several international conferences and is co-inventor of three patents. He has served as a referee for a number of journals. He has led a number of projects on national level in Bulgaria, as well as international bilateral projects. During 1999-2002, he served as a National (Bulgarian) coordinator of the Cost 268 initiative, and is currently the National coordinator for two ongoing COST actions: COST 288 and COST P11. He co-chaired the PHASE (PHysics and Applications of SEmiconductor LASERs), Supélec, Metz, France, and the PPCM (Physics of Photonics Crystal and Metamaterials), Brussels, international workshops, and acted as a Guest Editor of Special Issues of Optical and Quantum Electronics related to the workshops.

Dr. Panajotov is currently a member of OSA and SPIE. 